\title{
Streamline Diffusion Methods for the Incompressible Euler and Navier-Stokes Equations
}

\author{
By Claes Johnson and Jukka Saranen
}

\begin{abstract}
We present and analyze extensions of the streamline diffusion finite element method to the time-dependent two-dimensional Navier-Stokes equations for an incompressible fluid in the case of high Reynolds numbers. The limit case with zero viscosity, the Euler equations, is also considered.
\end{abstract}

Introduction. The Streamline Diffusion method is a finite element method for convection-dominated convection-diffusion problems recently introduced by Hughes and Brooks [5], [6] in the case of stationary problems. The mathematical analysis of this method for linear problems, together with extensions to time-dependent problems using space-time elements, was started in Johnson and Nävert [8] and was continued in [9], [16] and [10]. The outcome of this work is that the SD (Streamline Diffusion)-method can be demonstrated to have both good stability properties and high accuracy, a combination of desirable features not shared by previously known finite element methods; standard methods either (as the usual Galerkin method) are formally higher-order accurate, but lack in stability and produce severely oscillating solutions if the exact solution is nonsmooth, or (as the classical artificial viscosity or upwind method) contain a large amount of artificial diffusion, limiting the accuracy to at most first-order. The main theoretical results for the SD-method in [8], [9], [16] and [10] are almost-optimal error estimates, together with localization results which show that effects are propagated in the discrete problem in a way similar to what is the case in the continuous problem. In particular, it follows from these localization results that the presence of, e.g., a boundary layer in the exact solution only affects the accuracy of the discrete solution close to the layer. This is in contrast to the usual Galerkin method, where the presence of a boundary layer in general severely degrades the accuracy in the whole domain. The analysis of the SD-method also shows the necessity of sharpening the classical stability concept for finite element (or finite difference) methods for hyperbolic type problems, such as, e.g., convection-diffusion problems with dominating convection.

The purpose of this note is to present extensions of the SD-method to some nonlinear hyperbolic problems in fluid mechanics: The time-dependent two-dimensional Navier-Stokes equations for an incompressible Newtonian fluid in the case of

Received November 16, 1984; revised September 23, 1985. 1980 Mathematics Subject Classification. Primary 65N30.

Key words and phrases. Finite element method, incompressible flow, time-dependent, high Reynolds number.

(1986 American Mathematical Society $0025-5718 / 86 \$ 1.00+\$ .25$ per page 
high Reynolds number and also the limit case with zero viscosity, the Euler equations. We shall present methods for which global error estimates can be proved which are analogous to those mentioned above for linear problems. Propagation-ofinformation results for the discrete versions of the nonlinear problems considered would be very desirable to have, but seem very difficult to obtain; as far as we know, results of this nature are not even known for the continuous problems. For earlier work on SD-methods for the incompressible Navier-Stokes equations, see [7].

An outline of this note is as follows: In Section 1 we briefly review the SD-method for a linear convection-dominated convection-diffusion problem and recall the basic error estimate in this case. In Section 2 we present and analyze a streamline-diffusion type method for the Euler equations for an incompressible fluid in the case of two space dimensions. This method is based on using the stream function-vorticity formulation of the Euler equations. Finally, in Section 3 we consider two methods for the time-dependent Navier-Stokes equations also in two dimensions: One method using a velocity-pressure formulation, and one method using a velocity-pressure-vorticity formulation. The latter method is a streamline-diffusion variant of a mixed method for the Navier-Stokes equations previously analyzed by Girault and Raviart [4] in the case of low Reynolds numbers.

In the methods considered in this note, the approximate solution is sought on each time level as a piecewise polynomial on a finite element grid not necessarily aligned with the flow. We plan to compare our methods with 'grid free' so-called vortex methods, which have recently attracted renewed interest (see, e.g., [1]). Numerical experiments with the methods studied in the paper are under way and the results will be presented elsewhere.

In what follows, $C$ will denote a positive constant, not necessarily the same at each occurrence, independent of the parameters $h$ and $\varepsilon$.

\section{A Linear Convection-Diffusion Problem.}

1.1. The Continuous Problem. As a model for time-dependent convection-dominated convection-diffusion problems we shall consider the following problem: Find the scalar function $u=u(x, t)$ such that

$$
\left\{\begin{aligned}
u_{t}+u_{\beta}-\varepsilon \Delta u & =f & & \text { in } \Omega \times I, \\
u & =0 & & \text { on } \Gamma \times I, \\
u & =u_{0} & & \text { in } \Omega \text { for } t=0,
\end{aligned}\right.
$$

where $\Omega$ is a bounded domain in $\mathbf{R}^{2}$ with boundary $\Gamma, u_{t}=\partial u / \partial t, u_{\beta}=\beta \cdot \nabla u$, with $\nabla u$ the gradient with respect to $x=\left(x_{1}, x_{2}\right) \in \mathbf{R}^{2}$, and $\beta=\left(\beta_{1}, \beta_{2}\right)$ is a given smooth vector-field and $\varepsilon>0$ a small constant. Further, $f$ and $u_{0}$ are given data, and $I=(0, T)$ is a given time interval.

1.2. The Streamline-Diffusion Method. The SD-method for (1.1) is based on using finite elements over the space-time domain $Q=\Omega \times I$, i.e., a finite element formulation is used not only in space, but also to discretize in time. To define this method, let $\mathscr{T}_{h}=\{\tau\}$ be a finite element subdivision of $\Omega$ with elements $\tau$ and let $0=t_{0}<t_{1}<\cdots<t_{M}=T$ be a subdivision of the time interval $I$ into intervals $I_{m}=\left(t_{m}, t_{m+1}\right)$. Let $\mathscr{C}_{h}=\{K\}$ be the corresponding subdivision of $Q$ into elements $K=\tau \times I_{m}$ with $h$ representing the maximum of the diameters of the $K \in \mathscr{C}_{h}$, and 
let $P_{k}(K)$ be the set of polynomials in $x$ and $t$ of degree at most $k$ on $K$, and define for $k \geqslant 1$

$$
V_{h}=\left\{v \in \mathscr{H}:\left.v\right|_{K} \in P_{k}(\tau) \times P_{k}\left(I_{m}\right) \forall K=\tau \times I_{m} \in \mathscr{C}_{h}\right\},
$$

where $\mathscr{H}=\prod_{m=0}^{M-1} H^{1}\left(S_{m}\right)$, with $S_{m}=\Omega \times I_{m}$. In other words, $V_{h}$ is the set of piecewise polynomial functions on $\mathscr{C}_{h}$ of degree at most $k$ that are continuous in $x$ and possibly discontinuous in $t$ across the time levels $t_{m}, m=1, \ldots, M-1$. We shall assume that $\mathscr{C}_{h}$ is a regular subdivision of $Q$, i.e., for each $K \in \mathscr{C}_{h}$ there is an inscribed sphere in $K$ such that the ratio of the diameter of this sphere and the diameter of $K$ is bounded below, independently of $K$ and $h$.

We shall use the following notation: Given a domain $G$, let $(\cdot, \cdot)_{G}$ denote the usual $L_{2}(G)$ scalar product and $\|\cdot\|_{G}$ the corresponding norm. Also, $H^{s}(G)$ for $s$ a positive integer will denote the usual Sobolev space of functions with square integrable derivatives of order less than or equal to $s$ with norm $\|\cdot\|_{s, G}$. Further, for piecewise polynomials $v$ and $w$ defined on the triangulation $\mathscr{C}_{h}^{\prime}=\{K\}$, where $\mathscr{C}_{h}^{\prime} \subset \mathscr{C}_{h}$, and for differential operators $D_{i}$, we use the notation

$$
\left(D_{1} v, D_{2} w\right)_{Q^{\prime}}=\sum_{K \in \mathscr{C}_{h}^{\prime}}\left(D_{1} v, D_{2} w\right)_{K}, \quad Q^{\prime}=\bigcup_{K \in \mathscr{C}_{h}^{\prime}} K,
$$

i.e., we just sum the integrals over each element $K \in \mathscr{C}_{h}^{\prime}$. We also write

$$
\begin{aligned}
(w, v)_{m} & =(w, v)_{s_{m}}, \quad\|v\|_{m}=(v, v)_{m}^{1 / 2}, \\
\langle w, v\rangle_{m} & =\left(w\left(\cdot, t_{m}\right), v\left(\cdot, t_{m}\right)\right)_{\Omega}, \quad|v|_{m}=\langle v, v\rangle_{m}^{1 / 2}, \\
v_{ \pm}(x, t) & =\lim _{s \rightarrow 0^{ \pm}} v(x, t+s), \quad[v]=v_{+}-v_{-} .
\end{aligned}
$$

The SD-method for (1.1) can now be formulated as follows: Find $u^{h} \in \stackrel{\circ}{V}_{h}=$ $\left\{v \in V_{h}: v=0\right.$ on $\left.\Gamma \times I\right\}$ such that for $m=0, \ldots, M-1$,

$$
\begin{aligned}
\left(u_{t}^{h}+u_{\beta}^{h}, v+\delta\left(v_{t}+v_{\beta}\right)\right)_{m}+\left\langle\left[u^{h}\right], v_{+}\right\rangle_{m}+\varepsilon(\nabla u, \nabla v)_{m} & \\
& -\varepsilon \delta\left(\Delta u^{h}, v_{t}+v_{\beta}\right)_{m}=\left(f, v+\delta\left(v_{t}+v_{\beta}\right)\right)_{m} \quad \forall v \in \stackrel{V}{h}_{h},
\end{aligned}
$$

where $\delta=\bar{C} h$ with $\bar{C}$ a suitably chosen (sufficiently small, see [10] and below) positive constant and $u_{-}^{h}(\cdot, 0)=u_{0}$ are initial data and where, according to the above convention, the expression $\left(\Delta u^{h}, v_{t}+v_{\beta}\right)_{m}$ is interpreted as a sum of integrals over the elements $K \in \mathscr{C}_{h}$ in the slab $S_{m}$.

It is proved in [16] that if $u_{-}^{h}\left(\cdot, t_{m}\right)$ is given, then (1.3) defines $u^{h}$ uniquely in the slab $S_{m}$ and thus $u^{h}$ can be computed successively on the slabs $S_{m}$, starting at $S_{0}$ where $u_{-}^{h}(\cdot, 0)=u_{0}$ is given. For each $m,(1.3)$ is equivalent to a linear system of equations and thus (1.3) corresponds to an implicit scheme for (1.1).

Remark 1.1. If $\varepsilon>h$ we would choose $\delta=0$ and then (1.3), as regards the time-discretization, would coincide with the Discontinuous Galerkin method, recently analyzed in [3] in the case of a parabolic-type problem corresponding to choosing $\varepsilon \sim 1$ in (1.1) (see also [12]). In the parabolic case, the Discontinuous Galerkin method for time-discretization is demonstrated to have very satisfactory properties, resulting in a method of order $2 k+1$ with respect to $t$, when using polynomials of order $k$ in time. In fact, if $f \equiv 0$ and the time step is uniform, then the difference methods corresponding to the subdiagonal in the Padé table are retrieved in this way. 
Note that the feature that distinguishes (1.3) from conventional finite element or Galerkin methods is the presence of the term $\delta\left(v_{t}+v_{\beta}\right)$. The rationale for this modification is that choosing $v=u^{h} \in \stackrel{\circ}{V}_{h}$ in (1.3), and summing over $m$, we obtain control of a term of the form

$$
\left[\delta\left\|u_{t}^{h}+u_{\beta}^{h}\right\|_{Q}^{2}+\left|u_{-}^{h}\right|_{M}^{2}+\left|u_{+}^{h}\right|_{0}^{2}+\sum_{m=2}^{M-1}\left|\left[u^{h}\right]\right|_{m}^{2}\right]^{1 / 2},
$$

in terms of the data $u_{0}$ and $f$. This extra stability, as compared with Standard Galerkin, where $\delta=0$, is the key to the good properties of the method, allowing "almost optimal" error estimates and localization results to be demonstrated [10], [16]. The basic error estimate for (1.3), when $\varepsilon<h$, reads as follows,

$$
\left\|u-u^{h}\right\| \leqslant C h^{k+1 / 2}\|u\|_{k+1, Q}
$$

where

$$
\|w\|=\left[\max _{0 \leqslant t \leqslant T}\|w(\cdot, t)\|_{\Omega}^{2}+h\left\|w_{t}+w_{\beta}\right\|_{Q}^{2}+\varepsilon\|\nabla w\|_{Q}^{2}+\sum_{m=1}^{M-1} \|\left.[w]\right|_{m} ^{2}\right]^{1 / 2} .
$$

Remark 1.2. For the stationary analogue of (1.1) with no $u_{t}$-term, the modified test function in the SD-method has the form $\left(v+\delta v_{\beta}\right)$, which together with the term $u_{\beta}^{h}$ introduces a term of the form $\delta\left(u_{\beta}^{h}, v_{\beta}\right)$ which can be interpreted as resulting from diffusion $-\delta u_{\beta \beta}$ acting only in the streamline direction $\beta$. This is the motivation for the term "streamline diffusion". Note that in the time-dependent problem (1.1) it is important to introduce the term $\delta\left(u_{t}^{h}+u_{\beta}^{h}, v_{t}+v_{\beta}\right)$ corresponding to a diffusion acting in the direction $(\beta, 1)$ in space-time (cf. [6], [7], where only the modification $\left(v+\delta v_{\beta}\right)$ is used, even in the time-dependent case).

For numerical experiments with the SD-method (1.3), demonstrating the good properties of this method, also in the case of nonsmooth exact solutions, together with comparisons with the nonsatisfactory results obtained when putting $\delta=0$, we refer to [16].

1.3. Discontinuous Galerkin. If $\varepsilon=0$ in (1.1), then we can use trial functions in the discrete problem, which may be discontinuous across interelement boundaries also in the space variable. Note that with $\varepsilon=0,(1.1 \mathrm{~b}, \mathrm{c})$ is replaced by

$$
u=u_{0} \text { on } \partial Q_{-} \text {, }
$$

where

$$
\partial Q_{-}=\left\{(x, t) \in \partial Q: n_{t}(x, t)+n(x, t) \cdot \beta(x, t)<0\right\},
$$

and $u_{0}(x, t)=0$ if $t>0$. Here, of course, $\partial Q=\Omega \times\{0\} \cup \Omega \times\{T\} \cup \Gamma \times I$, and $\left(n, n_{t}\right)$ is the outward unit normal to $\partial Q$ in $\mathbf{R}^{3}$.

To define a finite element method using discontinuous functions, let us introduce the following notation: If $\beta=\left(\beta_{1}, \beta_{2}\right)$ is a given smooth vector field on $Q$, we define for $K \in \mathscr{C}_{h}$

$$
\partial K_{\mp}(\beta)=\left\{(x, t) \in \partial K: n_{t}(x, t)+n(x, t) \cdot \beta(x, t) \lessgtr 0\right\},
$$

where $\left(n, n_{t}\right)$ denotes the outward unit normal to $K \subset Q$. Further, let

$$
w_{\mp}(x, t)=\lim _{s \rightarrow 0^{\mp}} w(x+s \beta, t+s), \quad[w]=w_{+}-w_{-},
$$


and introduce for $k \geqslant 0$,

$$
W_{h}=\left\{v \in L_{2}(Q):\left.v\right|_{K} \in P_{k}(K) \forall K \in \mathscr{C}_{h}\right\}
$$

The Discontinuous Galerkin method for (1.1) can now be formulated as follows: Find $u^{h} \in W_{h}$ such that

$$
\begin{gathered}
\left(u_{t}^{h}+u_{\beta}^{h}, v+\delta\left(v_{t}+v_{\beta}\right)\right)_{Q}+\sum_{K \in \mathscr{C}_{h}} \int_{\partial K_{-}(\beta)}\left[u^{h}\right] v_{+}\left|n_{t}+n \cdot \beta\right| d s \\
=\left(f, v+\delta\left(v_{t}+v_{\beta}\right)\right)_{Q} \quad \forall v \in W_{h},
\end{gathered}
$$

where $\delta=0$ or $\delta=h$, and $u_{-}=u_{0}$ on $\partial Q_{-}$. With $\delta=0$, this is the usual Discontinuous Galerkin method, first analyzed in [13], where $L_{2}$-error estimates of order $\mathcal{O}\left(h^{k}\right)$ are proved. In [11], these estimates are improved to $\mathcal{O}\left(h^{k+1 / 2}\right)$, and it is demonstrated that the Discontinuous Galerkin method with $\delta=0$ or $\delta=h$ has properties very similar to the SD-method, in particular allowing the localization results for the SD-method to be carried over to the present case. Further, the Discontinuous Galerkin method (1.6) gives an explicit scheme, whereby the discrete solution on each slab $S_{m}$ can be computed successively on triangle after triangle, starting at the inflow boundary of $S_{m}$.

Remark 1.3. One may use in (1.3) or (1.6) different finite element subdivisions in different slabs $S_{m}$. This can be used, e.g., to locally refine the mesh around a progressing sharp front.

\section{The Euler Equations.}

2.1. The Continuous Problem. Let us now assume that $\Omega \subset \mathbf{R}^{2}$ is simply connected with smooth boundary $\Gamma$, and let us recall the Euler equations for an incompressible fluid: Given $g$ and $u_{0}$, find the velocity $u=\left(u_{1}, u_{2}\right)$ and the pressure $p$ such that

$$
\begin{array}{rlrl}
u_{t}+u \cdot \nabla u+\nabla p & =g & & \text { in } \Omega \times I, \\
\operatorname{div} u=0 & & \text { in } \Omega \times I, \\
u \cdot n=0 & & \text { on } \Gamma \times I, \\
u & =u_{0} & & \text { in } \Omega \text { for } t=0,
\end{array}
$$

where $n=n(x)$ is the outward unit normal to $\Gamma$. It is known (see, e.g., [17]) that if $g$ and $u_{0}$ are smooth, with $\operatorname{div} u_{0}=0$ in $\Omega$ and $u_{0} \cdot n=0$ on $\Gamma$, then (2.1) admits a unique smooth solution for any $T$.

Let us now reformulate (2.1) using the stream function-vorticity formulation. Since $\Omega$ is simply connected and $\operatorname{div} u=0$, there is a unique stream function $\psi(x, t)$ such that

$$
u=\operatorname{rot} \psi \equiv\left(\frac{\partial \psi}{\partial x_{2}},-\frac{\partial \psi}{\partial x_{1}}\right),\left.\quad \psi\right|_{\Gamma}=0 .
$$

Alternatively, $\psi(\cdot, t)$ can be specified as the unique solution of the Poisson equation

$$
\left\{\begin{aligned}
-\Delta \psi(\cdot, t) & =\omega(\cdot, t) & & \text { in } \Omega, \\
\psi & =0 & & \text { on } \Gamma,
\end{aligned}\right.
$$

where

$$
\omega=\operatorname{rot} u \equiv \frac{\partial u_{2}}{\partial x_{1}}-\frac{\partial u_{1}}{\partial x_{2}}
$$


is the vorticity of the velocity field $u$. Applying the operator rot to (2.1a), we obtain the following reformulation of (2.1): Find $\omega$ such that

$$
\left\{\begin{aligned}
\omega_{t}+u(\omega) \cdot \nabla \omega=f & \text { in } \Omega \times(0, T), \\
\omega=\omega_{0} & \text { in } \Omega \text { for } t=0,
\end{aligned}\right.
$$

where $f=\operatorname{rot} g, \omega_{0}=\operatorname{rot} u_{0}$, and $u(\omega)=\operatorname{rot} \psi$, where $\psi$ satisfies (2.2) for $0<t<T$. We see that (2.3) has formally the form (1.1) in the case $\varepsilon=0$ with a coefficient $\beta$ depending on $\omega$. Note that we do not have to specify any boundary condition in (2.3), since $u \cdot n=0$ on $\Gamma$.

2.2. SD With Trial Functions Continuous in Space. Let us now construct an SD-method for (2.3) following the pattern of Section 1.2 and using the same notation. Define for $k \geqslant 1$,

$$
\begin{aligned}
\dot{\Psi}_{h}=\{\phi \in \mathscr{H}: \phi \in & P_{k+1}(\tau) \times P_{k}\left(I_{m}\right) \\
& \left.\forall K=\tau \times I_{m} \in \mathscr{C}_{h}, \phi=0 \text { on } \Gamma \times I\right\},
\end{aligned}
$$

i.e., $\Psi_{h}$ consists of piecewise polynomials of degree $k+1$ and $k$ in $x$ and $t$, respectively, which are continuous in $x$ and discontinuous in $t$. The SD-method for (2.3) can now be formulated as follows: Find $\omega^{h} \in V_{h}$, with $V_{h}$ defined by (1.2), such that for $m=0,1,2, \ldots, M-1$,

$$
\begin{gathered}
\left(\omega_{t}^{h}+u^{h}\left(\omega^{h}\right) \cdot \nabla \omega^{h}, \theta+h\left(\theta_{t}+u^{h}\left(\omega^{h}\right) \cdot \nabla \theta\right)\right)_{m}+\left\langle\left[\omega^{h}\right], \theta_{+}\right\rangle_{m} \\
=\left(f, \theta+h\left(\theta_{t}+u^{h}\left(\omega^{h}\right) \cdot \nabla \theta\right)\right)_{m} \quad \forall \theta \in V_{h},
\end{gathered}
$$

where $u^{h}\left(\omega^{h}\right)=\operatorname{rot} \psi^{h}$ and $\psi^{h} \in \Psi_{h}$ satisfies

$$
\left(\nabla \psi^{h}, \nabla \phi\right)_{m}=\left(\omega^{h}, \phi\right)_{m} \quad \forall \phi \in \stackrel{\circ}{\Psi}_{h},
$$

and $\omega_{-}^{h}(\cdot, 0)=\omega_{0}$.

We now analyze this method and introduce the notation:

$$
\begin{gathered}
B(w ; v, \theta)=\sum_{m=0}^{M-1}\left(v_{t}+w \cdot \nabla v, \theta+h\left(\theta_{t}+u^{h}\left(\omega^{h}\right) \cdot \nabla \theta\right)\right)_{m} \\
\quad+\sum_{m=1}^{M-1}\left\langle[v], \theta_{+}\right\rangle_{m}+\left\langle v_{+}, \theta_{+}\right\rangle_{0}, \\
L(\theta)=\sum_{m=0}^{M-1}\left(f, \theta+h\left(\theta_{t}+u^{h}\left(\omega^{h}\right) \cdot \nabla \theta\right)\right)_{m}+\left\langle\omega_{0}, \theta_{+}\right\rangle_{0} .
\end{gathered}
$$

The problem (2.5) can then be formulated as follows: Find $\omega^{h} \in V_{h}$ such that

$$
B\left(u^{h}\left(\omega^{h}\right) ; \omega^{h}, \theta\right)=L(\theta) \quad \forall \theta \in V_{h},
$$

where $u^{h}\left(\omega^{h}\right)=\operatorname{rot} \psi^{h}$ and $\psi^{h} \in \stackrel{\circ}{\Psi}_{h}$ satisfies $(2.5 \mathrm{~b})$. We note that, since $u^{h}\left(\omega^{h}\right)=$ rot $\psi^{h}$, the normal component $u^{h}\left(\omega^{h}\right) \cdot n$ is continuous across interelement boundaries and $\operatorname{div} u^{h}\left(\omega^{h}\right) \equiv 0$ in each element $K$, so that

$$
\operatorname{div} u^{h}\left(\omega^{h}\right)=0 \quad \text { in } Q
$$

and also

$$
u^{h}\left(\omega^{h}\right) \cdot n=0 \text { on } \Gamma \times I \text {. }
$$


Below we shall refer to the following results, using the notation:

$$
\|\theta\|^{2}=\frac{1}{2}\left[|\theta|_{M}^{2}+|\theta|_{0}^{2}+\sum_{m=1}^{M-1}|[\theta]|_{m}^{2}+2 h\left\|\theta_{t}+u^{h}\left(\omega^{h}\right) \cdot \nabla \theta\right\|_{Q}^{2}\right] .
$$

LEMMA 2.1. We have

$$
B\left(u^{h}\left(\omega^{h}\right) ; \theta, \theta\right)=\|\theta\|^{2} \quad \forall \theta \in \mathscr{H} .
$$

Proof. The lemma follows easily from the definition of $B$, using the facts that by integration by parts and (2.7),

$$
\begin{gathered}
\left(\theta_{t}, \theta\right)+\sum_{m=1}^{M-1}\left\langle[\theta], \theta_{+}\right\rangle_{m}+\left\langle\theta_{+}, \theta_{+}\right\rangle_{0}=\frac{1}{2}\left[|\theta|_{M}^{2}+|\theta|_{0}^{2}+\sum_{m=1}^{M-1}|[\theta]|_{m}^{2}\right], \\
\left(u^{h}\left(\omega^{h}\right) \cdot \nabla \theta, \theta\right)_{Q}=0 .
\end{gathered}
$$

LEMMA 2.2. For any constant $C_{1}>0$, we have for $\theta \in \mathscr{H}$

$$
\|\theta\|_{Q}^{2} \leqslant\left[\frac{h}{C_{1}}\left\|\theta_{t}+u^{h}\left(\omega^{h}\right) \cdot \nabla \theta\right\|_{Q}^{2}+\sum_{m=1}^{M}\left|\theta_{-}\right|_{m}^{2} h\right] \exp \left(C_{1} h\right) .
$$

Proof. For $t_{m}<t<t_{m+1}$, we have using again (2.7),

$$
\begin{aligned}
\|\theta(t)\|_{\Omega}^{2} & =\left|\theta_{-}\right|_{m+1}^{2}-\int_{t}^{t_{m+1}} \frac{d}{d t}\|\theta(s)\|_{\Omega}^{2} d s \\
& =\left|\theta_{-}\right|_{m+1}^{2}-2 \int_{t}^{t_{m+1}}\left(\theta_{t}+u^{h}\left(\omega^{h}\right) \cdot \nabla \theta, \theta\right)_{\Omega} d s \\
& \leqslant\left|\theta_{-}\right|_{m+1}^{2}+\frac{1}{C_{1}}\left\|\theta_{t}+u^{h}\left(\omega^{h}\right) \cdot \nabla \theta\right\|_{m}^{2}+C_{1} \int_{t}^{t_{m+1}}\|\theta(s)\|_{\Omega}^{2} d s,
\end{aligned}
$$

so that by Gronwall's inequality, for $t_{m}<t<t_{m+1}$,

$$
\|\theta(t)\|_{\Omega}^{2} \leqslant\left[\frac{1}{C_{1}}\left\|\theta_{t}+u^{h}\left(\omega^{h}\right) \cdot \nabla \theta\right\|_{m}^{2}+\left|\theta_{-}\right|_{m+1}^{2}\right] \exp \left(C_{1} h\right) .
$$

Integrating over $t_{m}<t<t_{m+1}$ and then summing over $m=0, \ldots, M-1$, we obtain the desired result.

LEMMA 2.3. There is a constant $C$ such that if $\hat{\psi}^{h} \in \dot{\Psi}_{h}$ satisfies

$$
\left(\nabla \hat{\psi}^{h}, \nabla \phi\right)_{Q}=(\omega, \phi)_{Q} \quad \forall \phi \in \dot{\Psi}_{h},
$$

then

$$
\left\|u(\omega)-u^{h}\left(\omega^{h}\right)\right\|_{Q} \leqslant C h^{k+1}\|\psi\|_{k+2, Q}^{*},
$$

where $\psi$ satisfies $(2.2), u(\omega)=\operatorname{rot} \psi, u^{h}(\omega)=\operatorname{rot} \hat{\psi}^{h}$ and

$$
\|\psi\|_{k+2, Q}^{*}=\left(\int_{I}\left[\|\psi(\cdot, s)\|_{k+2, \Omega}^{2}+\left\|\frac{\partial^{k+1}}{\partial s^{k+1}} \psi(\cdot, s)\right\|_{1, \Omega}^{2}\right] d s\right)^{1 / 2} .
$$

Proof. We have, since $\left(\nabla \psi-\nabla \hat{\psi}^{h}, \nabla \phi\right)_{Q}=0$ for all $\phi \in \stackrel{\circ}{\Psi}^{h}$,

$$
\left\|\nabla \psi-\nabla \hat{\psi}^{h}\right\|_{Q} \leqslant\|\nabla \psi-\nabla \phi\|_{Q} \quad \forall \phi \in \stackrel{\dot{\Psi}}{ }^{h} .
$$

Here we choose $\phi=R \otimes P \psi$, where

$$
(R \otimes P \psi)(x, t)=R v(x, \cdot)(t), \quad v(x, s)=(P \psi(\cdot, s))(x),
$$


and where

$$
P: \stackrel{\circ}{H}^{1}(\Omega) \rightarrow \stackrel{\Phi}{\Phi}^{h}=\left\{\phi \in \stackrel{\circ}{H}^{1}(\Omega):\left.\phi\right|_{\tau} \in P_{k+1}(\tau) \forall \tau \in \mathscr{T}_{h}\right\}
$$

is the usual $\stackrel{\circ}{H}^{1}(\Omega)$-projection, and

$$
R: L^{2}\left(I_{m}\right) \rightarrow P_{k}\left(I_{m}\right)
$$

is the $L^{2}\left(I_{m}\right)$-projection. We have, with $v$ given by (2.9),

$$
\nabla(\psi-\phi)=\nabla(\psi-v)+\nabla(v-\phi) .
$$

Now, using standard estimates for $P$, we have for the first term

$$
\int_{\Omega}|\nabla(\psi(x, s)-P \psi(\cdot, s)(x))|^{2} d x \leqslant C h^{2(k+1)}\|\psi(\cdot, s)\|_{k+2, \Omega}^{2},
$$

so that by integration

$$
\|\nabla(\psi-v)\|_{Q} \leqslant C h^{k+1}\|\psi\|_{k+2, Q}^{*}
$$

Further, by the obvious commutation relations

$$
\nabla R=R \nabla, \quad \frac{\partial^{j}}{\partial s^{j}} P=P \frac{\partial^{j}}{\partial s^{j}},
$$

we have for the second term from standard estimates for $R$ and $P$ :

$$
\begin{aligned}
\int_{\Omega} \int_{I_{m}} \mid \nabla & \left.(v-\phi)\right|^{2} d t d x=\int_{\Omega} \int_{I_{m}}|\nabla v(x, s)-R(\nabla v(x, \cdot))(s)|^{2} d x d t \\
& \leqslant C h^{2(k+1)} \int_{\Omega} \int_{I_{m}}\left|\frac{\partial^{k+1}}{\partial s^{k+1}} \nabla v(x, s)\right|^{2} d s d x \\
& =C h^{2(k+1)} \int_{\Omega} \int_{I_{m}}\left|\nabla \frac{\partial^{k+1}}{\partial s^{k+1}} v(x, s)\right|^{2} d s d x \\
& =C h^{2(k+1)} \int_{I_{m}} \int_{\Omega}\left|\nabla\left(P \frac{\partial^{k+1}}{\partial s^{k+1}} \psi(\cdot, s)(x)\right)\right|^{2} d x d s \\
& \leqslant C h^{2(k+1)} \int_{I_{m}} \int_{\Omega}\left|\nabla \frac{\partial^{k+1}}{\partial s^{k+1}} \psi(x, s)\right|^{2} d x d s .
\end{aligned}
$$

Summation over $m$ then completes the proof.

LEMMA 2.4. For any $h>0$, the problem (2.6) admits at least one solution if $f \in L_{2}(Q)$ and $\omega_{0} \in L_{2}(\Omega)$.

Proof. We shall apply a variant of Brouwer's fixed point theorem to prove that, given $u_{-}^{h}\left(\cdot, t_{m}\right)$, the problem $(2.5)$ admits a solution for any $m$. Let $V^{m}=\left\{\left.v\right|_{I_{m}}\right.$ : $\left.v \in V_{h}\right\}$ and $\Psi^{m}=\left\{\left.\phi\right|_{I_{m}}: \phi \in \Psi_{h}\right\}$, and define $P^{m}: V^{m} \rightarrow V^{m}$ by

$$
\begin{aligned}
{\left[P^{m} v, \theta\right]=} & \left(v_{t}+u^{m}(v) \cdot \nabla v, \theta+h\left(\theta_{t}+u^{m}(v) \cdot \nabla \theta\right)\right)_{m} \\
& +\left\langle v_{+}, \theta_{+}\right\rangle_{m}-\left\langle u_{-}^{h}, \theta_{+}\right\rangle_{m} \\
& -\left(f, \theta+h\left(\theta_{t}+u^{m}(v) \cdot \nabla \theta\right)\right)_{m} \quad \forall \theta \in V^{m},
\end{aligned}
$$

where $u^{m}(v)=\operatorname{rot} \psi^{m}$, with $\psi^{m} \in \Psi^{m}$ satisfying

$$
\left(\nabla \psi^{m}, \nabla \phi\right)_{m}=(v, \phi)_{m} \quad \forall \phi \in \dot{\Psi}^{m}
$$


and

$$
[v, \theta]=\left\langle v_{-}, \boldsymbol{\theta}_{-}\right\rangle_{m+1}+\left\langle v_{+}, \boldsymbol{\theta}_{+}\right\rangle_{m}+(v, \boldsymbol{\theta})_{m} \text {. }
$$

Clearly, $P^{m}$ is well-defined and is continuous on $V^{m}$ (for a fixed $h$ ), with the norm $|\cdot|$ given by the scalar product $[\cdot, \cdot]$. Further, $P^{m} v \equiv 0$ if and only if $v=\omega^{h}$ satisfies (2.5). Now

$$
\begin{aligned}
{\left[P^{m} v, v\right]=} & \frac{1}{2}\left[\left|v_{-}\right|_{m+1}^{2}+\left|v_{+}\right|_{m}^{2}+2 h\left\|v_{t}+u^{m}(v) \cdot \nabla v\right\|_{m}^{2} \mid\right. \\
& -\|f\|_{m}\left(\|v\|_{m}+h\left\|v_{t}+u^{m}(v) \cdot \nabla v\right\|_{m}\right)-\left|u_{-}^{h}\right|_{m}\left|v_{+}\right|_{m},
\end{aligned}
$$

so that using Lemma 2.2,

$$
\left[P^{m} v, v\right] \geqslant C|v|^{2}-C|v|\left(\|f\|_{m}+\left|u_{-}^{h}\right|_{m}\right) .
$$

Hence, $\left[P^{m} v, v\right]>0$ if $|v|=r$ is large enough, and by Brouwer's fixed point theorem it follows (see [15]) that there exists $v=\omega^{m} \in V^{m}$ with $|v| \leqslant r$ such that $P v \equiv 0$, and the proof is complete.

For a uniqueness result we refer to the Appendix.

We now turn to error estimates. We write $e \equiv \omega-\omega^{h}=\eta-\zeta$, where $\eta=\omega-\tilde{\omega}^{h}$, $\zeta=\omega^{h}-\tilde{\omega}^{h}$, and $\tilde{\omega}^{h} \in V_{h}$ is a suitable interpolant of the exact solution $\omega$. Since $\omega$ satisfies (2.3), we have for $\theta \in V_{h}$

$$
B(u(\omega) ; \omega, \theta)=L(\theta),
$$

so that by (2.6) and Lemma 2.1,

$$
\begin{aligned}
\|\zeta\|^{2} & =B\left(u^{h}\left(\omega^{h}\right) ; \omega^{h}-\tilde{\omega}^{h}, \zeta\right)=B(u(\omega) ; \omega, \zeta)-B\left(u^{h}\left(\omega^{h}\right) ; \tilde{\omega}^{h}, \zeta\right) \\
& =B\left(u^{h}\left(\omega^{h}\right) ; \eta, \zeta\right)+\left[B(u(\omega) ; \omega, \zeta)-B\left(u^{h}\left(\omega^{h}\right) ; \omega, \zeta\right)\right] \\
& =T_{1}+T_{2},
\end{aligned}
$$

with the obvious definitions of $T_{1}$ and $T_{2}$. By integration by parts, using (2.7), we find

$$
\begin{aligned}
&\left|T_{1}\right|=\mid-\left(\eta, \zeta_{t}+u^{h}\left(\omega^{h}\right) \cdot \nabla \zeta\right)_{Q}+\left\langle\eta_{-}, \zeta_{-}\right\rangle_{M}-\sum_{m=1}^{M-1}\left\langle\eta_{-},[\zeta]\right\rangle_{m} \\
&+h\left(\eta_{t}+u^{h}\left(\omega^{h}\right) \cdot \nabla \eta, \zeta_{t}+u^{h}\left(\omega^{h}\right) \cdot \nabla \zeta\right)_{Q} \mid \\
& \leqslant \frac{1}{8}\|\zeta\|^{2}+C\left(h^{-1}\|\eta\|_{Q}^{2}+\sum_{m=1}^{M}\left|\eta_{-}\right|_{m}^{2}+h\left\|\eta_{t}+u^{h}\left(\omega^{h}\right) \cdot \nabla \eta\right\|_{Q}^{2}\right) .
\end{aligned}
$$

Further, by Lemma 2.3 we have

$$
\left\|u^{h}(\omega)-u(\omega)\right\|_{Q} \leqslant C h^{k+1}\|\psi\|_{k+2, Q}^{*}
$$

where $u^{h}(\omega)=\operatorname{rot} \hat{\psi}^{h}$ and $\hat{\psi}^{h} \in \Psi^{h}$ satisfies (2.8). Also,

$$
\left\|u^{h}\left(\omega^{h}\right)-u^{h}(\omega)\right\|_{Q} \leqslant C\left\|\omega-\omega^{h}\right\|_{Q} \leqslant C\left(\|\zeta\|_{Q}+\|\eta\|_{Q}\right),
$$

and hence, letting $\|\cdot\|_{\infty}$ denote the $L_{\infty}(Q)$-norm,

$$
\begin{aligned}
\left\|\eta_{t}+u^{h}\left(\omega^{h}\right) \cdot \nabla \eta\right\|_{Q} \leqslant & \left\|\eta_{t}+u(\omega) \cdot \nabla \eta\right\|_{Q}+\left\|\left(u^{h}(\omega)-u(\omega)\right) \cdot \nabla \eta\right\|_{Q} \\
& +\left\|\left(u^{h}(\omega)-u^{h}\left(\omega^{h}\right)\right) \cdot \nabla \eta\right\|_{Q} \\
\leqslant & \left\|\eta_{t}\right\|_{Q}+\|u(\omega)\|_{\infty}\|\nabla \eta\|_{Q} \\
& +\|\nabla \eta\|_{\infty}\left(\|\zeta\|_{Q}+\|\eta\|_{Q}+C h^{k+1}\|\psi\|_{k+2 . Q}^{*}\right) .
\end{aligned}
$$


Next,

$$
\begin{aligned}
T_{2}= & \left(\left(u(\omega)-u^{h}\left(\omega^{h}\right)\right) \cdot \nabla \omega, \zeta\right)_{Q} \\
& +h\left(\left(u(\omega)-u^{h}\left(\omega^{h}\right)\right) \cdot \nabla \omega, \zeta_{t}+u^{h}\left(\omega^{h}\right) \cdot \nabla \zeta\right)_{Q},
\end{aligned}
$$

so that by (2.12) and (2.13),

$$
\begin{aligned}
\left|T_{2}\right| \leqslant & C\|\nabla \omega\|_{\infty}\|\zeta\|_{Q}\left(\|\zeta\|_{Q}+\|\eta\|_{Q}+C h^{k+1}\|\psi\|_{k+2, Q}^{*}\right) \\
& +\frac{1}{8}\|\zeta\|^{2}+C h\left[\|\nabla \omega\|_{\infty}\left(\|\zeta\|_{Q}+\|\eta\|_{Q}+C h^{k+1}\|\psi\|_{k+2, Q}^{*}\right)\right]^{2} .
\end{aligned}
$$

We now assume that

$$
\|\nabla \omega\|_{\infty}+\|\nabla \eta\|_{\infty}+\|\omega\|_{k+1, Q} \leqslant C .
$$

In particular, this means by Sobolev imbedding and elliptic regularity, that also

$$
\|u(\omega)\|_{\infty}+\|\psi\|_{k+2, Q}^{*} \leqslant C .
$$

Combining now (2.10), (2.11), (2.14)-(2.16) and Lemma 2.1 with $C_{1}$ large enough, we get

$$
\|\zeta\|^{2} \leqslant C\left(h^{-1}\|\eta\|_{Q}^{2}+\sum_{m=1}^{M}\left|\eta_{-}\right|_{m}^{2}+h\|\eta\|_{1, Q}^{2}+\sum_{m=1}^{M}\left|\zeta_{-}\right|_{m}^{2} h+C h^{2 k+2}\right) .
$$

Finally, by standard interpolation theory, we have (see, e.g., [2]):

$$
\left[\|\eta\|_{Q}^{2}+h \sum_{m=1}^{M}\left|\eta_{-}\right|_{m}^{2}+h^{2}\|\eta\|_{1, Q}^{2}\right]^{1 / 2} \leqslant C h^{k+1}\|\omega\|_{k+1, Q} .
$$

We can now finish the argument by applying a discrete Gronwall inequality of the following form: Suppose $\left\{a_{k}\right\}_{1}^{M}$ satisfies, for $k=1, \ldots, M$ with $M=C_{3} / h$,

$$
a_{k} \leqslant C_{1} \sum_{1 \leqslant j \leqslant k} a_{j} h+C_{2}
$$

then there is a constant $C$, depending only on the constants $C_{i}$, such that for $h \leqslant 1 / 2 C_{1}$ we have $a_{k} \leqslant C, k=1, \ldots, M$.

We then obtain the following error estimate:

THEOREM 2.1. If the exact solution $\omega$ of (2.3) satisfies (2.16), and $\omega^{h} \in V_{h}$ satisfies (2.6), then $\left\|\omega-\omega^{h}\right\| \leqslant C h^{k+1 / 2}$.

Remark 2.1. The $\mathcal{O}\left(h^{k+1}\right)$-error from the approximate solution of the Poisson equation (2.2) according to (2.12) is here not fully balanced with the global $\mathcal{O}\left(h^{k+1 / 2}\right)$ estimate. To obtain such a balance one might in practice use a coarser mesh in $x$ for the Poisson equation (2.2), keeping the degree $k+1$ in $x$ for $\psi$, or one might instead reduce this degree to $k$ and mildly refine the mesh in $x$.

Remark 2.2. By Lemma 2.2 and the definition of the $\||\cdot| \mid-$-norm, we have from Theorem 2.1 in particular

$$
\max _{t \in I}\left\|\omega(\cdot, t)-\omega^{h}(\cdot, t)\right\|_{\Omega} \leqslant C h^{k+1 / 2},
$$

where $\omega^{h}\left(\cdot, t_{m}\right)=\omega_{ \pm}^{h}\left(\cdot, t_{m}\right)$. 
2.3. Discontinuous Galerkin. Extending the Discontinuous Galerkin method of Section 1.3 to the Euler equations, using the same notation, we obtain the following method: Find $\omega^{h} \in W_{h}$ such that

$$
\begin{gathered}
\left(\omega_{t}^{h}+\beta \cdot \nabla \omega^{h}, \theta+h\left(\theta_{t}+\beta \cdot \nabla \theta\right)\right)_{Q}+\sum_{K} \int_{\partial K_{-}(\beta)}\left[\omega^{h}\right] \theta_{+}\left|n_{t}+n \cdot \beta\right| d s \\
=\left(f, \theta+h\left(\theta_{t}+\beta \cdot \nabla \theta\right)\right)_{Q} \quad \forall \theta \in W_{h},
\end{gathered}
$$

where $\beta \equiv u^{h}\left(\omega^{h}\right)=\operatorname{rot} \psi^{h}$ and $\psi^{h} \in \stackrel{\circ}{\Psi}_{h}$ satisfies

$$
\left(\nabla \psi^{h}, \nabla \phi\right)_{Q}=\left(\omega^{h}, \phi\right)_{Q} \quad \forall \phi \in \stackrel{\circ}{\Psi}_{h},
$$

and $\omega_{-}^{h}(\cdot, 0)=\omega_{0}$. Recall that, since $\beta=\operatorname{rot} \psi^{h}$, we have that $n \cdot \beta$ is continuous across the interelement boundaries of $\mathscr{C}_{h}$, and thus $\partial K_{\mp}(\beta)$ is well-defined. Also, $\beta \cdot n=0$ on $\Gamma \times I$. To write (2.17) in more compact form, let us introduce the notation

$$
\begin{aligned}
B(w ; v, \theta)= & \left(v_{t}+\omega \cdot \nabla w, \theta+h\left(\theta_{t}+\beta \cdot \nabla \theta\right)\right)_{Q} \\
& +\sum_{K} \int_{\partial K_{-}(\beta)^{\prime}}[v] \theta_{+}\left|n_{t}+n \cdot \beta\right| d s+\left\langle v_{+}, \theta_{+}\right\rangle_{0}, \\
L(v)= & \left(f, \theta+h\left(\theta_{t}+\beta \cdot \nabla \theta\right)\right)_{Q}+\left\langle\omega_{0}, \theta_{+}\right\rangle_{0},
\end{aligned}
$$

where again $\beta=u^{h}\left(\omega^{h}\right)$ and $\partial K_{-}(\beta)^{\prime}=\partial K_{-}(\beta) \backslash \Omega \times\{0\}$. Then (2.17) can be written, with $u^{h}\left(\omega^{h}\right)=\operatorname{rot} \psi^{h}$ and $\psi^{h} \in \stackrel{\sim}{\Psi}_{h}$ satisfying (2.5b), in the form

$$
B\left(u^{h}\left(\omega^{h}\right) ; \omega^{h}, \theta\right)=L(\theta) \quad \forall \theta \in W_{h} .
$$

This method can be analyzed using arguments very similar to those of the previous subsection. In particular, we have the following analogues of Lemmas 2.1 and 2.2, with similar proofs (as above, $\beta=u^{h}\left(\omega^{h}\right)$ ).

LEMMA 2.5. We have, with $B$ defined by (2.18),

$$
B\left(u^{h}\left(\omega^{h}\right) ; \theta, \theta\right)=\|\theta\|^{2} \quad \forall \theta \in W_{h},
$$

where

$$
\|\theta\|^{2}=\frac{1}{2}\left[|\theta|_{M}^{2}+|\theta|_{0}^{2}+\sum_{K} \int_{\partial K_{-}(\beta)^{\prime}}[\theta]^{2}\left|n_{t}+n \cdot \beta\right| d s+2 h\left\|\theta_{t}+\beta \cdot \nabla \theta\right\|_{Q}^{2}\right] .
$$

LEMMA 2.6. For any constant $C_{1}>0$, we have for $\theta \in W_{h}$

$$
\begin{aligned}
\|\theta\|_{Q}^{2} \leqslant\left[\frac{h}{C_{1}} \| \theta_{t}+\beta \cdot\right. & \nabla \theta \|_{Q}^{2}+\sum_{m=1}^{M}\left|\theta_{-}^{2}\right|_{m} h \\
& \left.+h \sum_{K} \int_{\partial K_{-.}(\beta)^{\prime \prime}}[\theta]^{2}|n \cdot \beta| d s\right] \exp \left(C_{1} h\right),
\end{aligned}
$$

where $\partial K_{-}(\beta)^{\prime \prime}=\left\{(x, t) \in \partial K_{-}(\beta)^{\prime}: n_{t}(x, t)=0\right\}$.

The only difference in the analysis of (2.19), as compared to the above analysis of (2.6), is the appearance on the right-hand side of a term of the form

$$
\sum_{K} \int_{\partial K_{-}(\beta)^{\prime \prime}}[\zeta] \eta_{+}|n \cdot \beta| d s,
$$


where as above, $\zeta=\omega^{h}-\tilde{\omega}^{h}, \eta=\omega-\tilde{\omega}^{h}$ and $\beta=u^{h}\left(\omega^{h}\right)$. This means that we will have to bound a term of the form

$$
T_{3}=\sum_{K} \int_{\partial K_{-}(\beta)^{\prime \prime}}\left|\eta_{+}\right|^{2}\left|n \cdot u^{h}\left(\omega^{h}\right)\right| d s,
$$

which can be done as follows:

$$
\begin{aligned}
\left|T_{3}\right| & \leqslant\|\eta\|_{\infty}^{2} \sum_{K}\left[\int_{\partial K_{-}(\beta)^{\prime \prime}}\left|n \cdot u^{h}\left(\omega^{h}\right)\right|^{2} d s+\int_{\partial K_{-}(\beta)^{\prime \prime}} d s\right] \\
& \leqslant C\|\eta\|_{\infty}^{2} \sum_{K}\left[C h^{-1}\left\|u^{h}\left(\omega^{h}\right)\right\|_{K}^{2}+C h^{2}\right] \\
& \leqslant C h^{2 k+1}\|\omega\|_{k+1, \infty}^{2}\left(\|\zeta\|_{Q}^{2}+C\right),
\end{aligned}
$$

where we have used the facts that

$$
\int_{\partial K} v^{2} d s \leqslant C h^{-1} \int_{K} v^{2} d x \quad \forall v \in P_{k}(K),
$$

and that by (2.12) and (2.13),

$$
\left\|u^{h}\left(\omega^{h}\right)\right\|_{Q} \leqslant\|u(\omega)\|_{Q}+\|\eta\|_{Q}+\|\zeta\|_{Q}+C .
$$

Here and below, $\|\cdot\|_{k+1, \infty}$ denotes the $W_{\infty}^{k+1}(Q)$-norm. This leads to the following error estimate for (2.19), if $\omega \in W_{\infty}^{k+1}(Q)$ :

$$
\left\|\omega-\omega^{h}\right\| \leqslant C h^{k+1 / 2} .
$$

\section{The Navier-Stokes Equations.}

3.1. SD-Methods for Euler's Equation in $(u, p)$-formulation. It is not clear how to generalize the methods for the Euler equations of the previous section to the Navier-Stokes equations, the reason being that we do not have any boundary conditions for the vorticity. We shall therefore first present some different methods for the Euler equations, using the $(u, p)$-formulation $(2.1)$, which may naturally be extended to the Navier-Stokes case.

We first assume that the discrete velocities are continuous in $x$, and as before, satisfy the incompressibility condition exactly, i.e., we let the velocity space $W_{h}$ be given by

$$
W_{h}=\left\{\operatorname{rot} \phi: \phi \in \stackrel{\circ}{\Psi}_{h}\right\}
$$

where $\dot{\Psi}_{h}$, as before, is given by (2.4) and in addition satisfies

$$
\dot{\Psi}_{h} \subset \prod_{m=0}^{M-1} C^{1}\left(S_{m}\right) \text {. }
$$

Introducing also the pressure space

$$
Q_{h}=\left\{q \in \mathscr{H}:\left.q\right|_{K} \in P_{k}(\tau) \times P_{k}\left(I_{m}\right) \forall K=\tau \times I_{m} \in \mathscr{C}_{h}\right\},
$$

we now formulate the following SD-method for the Euler equations (2.1): Find $\left(u^{h}, p^{h}\right) \in W_{h} \times Q_{h}$ such that

$$
\begin{aligned}
\left(u_{t}^{h}\right. & \left.+u^{h} \cdot \nabla u^{h}+\nabla p^{h}, v+h\left(v_{t}+u^{h} \cdot \nabla v+\nabla q\right)\right)_{Q}+\sum_{m=0}^{M-1}\left\langle\left[u^{h}\right], v_{+}\right\rangle_{m} \\
& =\left(g, v+h\left(v_{t}+u^{h} \cdot \nabla v+\nabla q\right)\right)_{Q} \quad \forall(v, q) \in W_{h} \times Q_{h},
\end{aligned}
$$


where $u_{-}^{h}(\cdot, 0)=u_{0}$. Using the notation

$$
\begin{aligned}
& B(w ; \hat{v}, \hat{\phi})=\left(v_{t}+w \cdot \nabla v+\nabla q, \phi+h\left(\phi_{t}+u^{h} \cdot \nabla \phi+\nabla r\right)\right)_{Q} \\
&+\sum_{m=1}^{M-1}\left\langle[v], \phi_{+}\right\rangle_{m}+\left\langle v_{+}, \phi_{+}\right\rangle_{0}, \quad \hat{v}=(v, q), \hat{\phi}=(\phi, r), \\
& L(\hat{\phi})=\left(g, \phi+h\left(\phi_{t}+u^{h} \cdot \nabla \phi+\nabla r\right)\right)_{Q},
\end{aligned}
$$

we can formulate problem (3.4) as follows: Find $\hat{u}^{h}=\left(u^{h}, p^{h}\right) \in W_{h} \times Q_{h}$ such that

$$
B\left(u^{h} ; \hat{u}^{h}, \hat{v}\right)=L(\hat{v}) \quad \forall \hat{v} \in W_{h} \times Q_{h} .
$$

Note that the use of the modified test function $v+h\left(v_{t}+u^{h} \cdot \nabla v+\nabla q\right)$ will give control over the quantity $\sqrt{h}\left\|u_{t}^{h}+u^{h} \cdot \nabla u^{h}+\nabla p^{h}\right\|_{Q}$ in (3.6). It seems difficult to obtain control over the quantity $\sqrt{h}\left\|u_{t}^{h}+u^{h} \cdot \nabla u^{h}\right\|_{Q}$, since multiplication with $\left(v+h\left(v_{t}+u^{h} \cdot \nabla v\right)\right)$ will introduce a term $\left(\nabla p^{h}, u^{h} \cdot \nabla v\right)_{Q}$, which will not vanish in general and seems difficult to bound. Note further that taking $v=0$ in (3.4), we obtain the following equation for $p^{h} \in Q_{h}$ :

$$
\left(\nabla p^{h}, \nabla q\right)_{Q}=\left(g-u_{t}^{h}-u^{h} \cdot \nabla u^{h}, \nabla q\right) \quad \forall q \in Q_{h},
$$

which corresponds to a Poisson equation with Neumann boundary conditions for the pressure.

We now analyze (3.6) and introduce the norm

$$
\|\hat{v}\|^{2}=\frac{1}{2}\left[|v|_{M}^{2}+|v|_{0}^{2}+\sum_{m=1}^{M-1}|[v]|_{m}^{2}+2 h\left\|v_{t}+u^{h} \cdot \nabla v+\nabla q\right\|_{Q}^{2}\right] .
$$

As above (cf. Lemma 2.1), it follows that

$$
B\left(u^{h}, \hat{v}, \hat{v}\right)=\|\hat{v}\|^{2} \quad \forall \hat{v} \in W_{h} \times Q_{h},
$$

and we also have the following analogue of Lemma 2.2.

LEMMA 3.1. For any constant $C_{1}>0$ we have for $\hat{v} \in W_{h} \times Q_{h}$ :

$$
\|v\|_{Q}^{2} \leqslant\left[\frac{h}{C_{1}}\left\|v_{t}+u^{h} \cdot \nabla v+\nabla q\right\|_{Q}^{2}+\sum_{m=1}^{M}\left|v_{-}\right|_{m}^{2} h\right] \exp \left(C_{1} h\right) .
$$

Proof. We have, for $t_{m}<t<t_{m+1}$,

$$
\begin{aligned}
\|v(t)\|_{\Omega}^{2} & =\left|v_{-}\right|_{m+1}^{2}-\int_{t}^{t_{m+1}} \frac{d}{d t}\|v(s)\|_{\Omega}^{2} d s \\
& =\left|v_{-}\right|_{m+1}^{2}-2 \int_{t}^{t_{m+1}}\left(v_{t}+u^{h} \cdot \nabla v+\nabla q, v\right)_{\Omega} d s \\
& \leqslant\left|v_{-}\right|_{m+1}^{2}+\frac{1}{C_{1}}\left\|v_{t}+u^{h} \cdot \nabla v+\nabla q\right\|_{m}^{2}+C_{1} \int_{t}^{t_{m+1}}\|v(s)\|_{\Omega}^{2} d s,
\end{aligned}
$$

from which the desired result follows, arguing as in the proof of Lemma 2.2.

Further, existence of a solution of (3.6) can be proved using basically the argument of the proof of Lemma 2.4.

We now prove the following error estimate.

THEOREM 3.1. If the solution $(u, p)$ of $(2.1)$ satisfies

$$
\|u\|_{k+1, Q}+\|p\|_{k+1, Q}+\|u\|_{1, \infty} \leqslant C,
$$


and $\left(u^{h}, p^{h}\right) \in W_{h} \times Q_{h}$ satisfies (3.4), then

$$
\max _{t \in I}\left\|u(\cdot, t)-u^{h}(\cdot, t)\right\|_{\Omega}+\left\|u-u^{h}\right\| \leqslant C h^{k+1 / 2} .
$$

Proof. We write

$$
\begin{aligned}
\hat{u}-\hat{u}^{h} & =(\hat{u}-\hat{w})+\left(\hat{w}-\hat{u}^{h}\right) \equiv \hat{\eta}+\hat{\zeta} \\
& \equiv(u-\tilde{u}, p-\tilde{p})+\left(\tilde{u}-u^{h}, \tilde{p}-p^{h}\right) \equiv(\eta, p-\tilde{p})+\left(\zeta, \tilde{p}-p^{h}\right),
\end{aligned}
$$

where $\hat{w}=(\tilde{u}, \tilde{p}) \in W_{h} \times Q_{h}$ is the interpolant of $\hat{u}=(u, p)$. Using the fact that

$$
B(u ; \hat{u}, \hat{v})=L(\hat{v}) \quad \forall \hat{v}=(v, q) \in W_{h} \times Q_{h},
$$

we have by (3.6),

$$
\begin{aligned}
\|\hat{\zeta}\|^{2} & =B\left(u^{h} ; \hat{u}^{h}-\hat{w}, \hat{\zeta}\right)=B(u ; \hat{u}, \hat{\zeta})-B\left(u^{h} ; \hat{w}, \hat{\zeta}\right) \\
& =B\left(u^{h} ; \hat{\eta}, \hat{\zeta}\right)+\left(B(u ; \hat{u}, \hat{\zeta})-B\left(u^{h} ; \hat{u}, \hat{\zeta}\right)\right) \\
& \equiv T_{1}+T_{2},
\end{aligned}
$$

with the obvious definition of $T_{1}$ and $T_{2}$. Integrating by parts, we have by standard interpolation estimates,

$$
\begin{aligned}
& \left|T_{1}\right|=\mid-\left(\eta, \zeta_{t}+u^{h} \cdot \nabla \zeta+\nabla\left(\tilde{p}-p^{h}\right)\right)_{Q}+\left\langle\eta_{-}, \zeta_{-}\right\rangle_{M} \\
& \quad-\sum_{m=1}^{M-1}\left\langle\eta_{-},[\zeta]\right\rangle_{m}+h\left(\eta_{t}+u^{h} \cdot \nabla \eta+\nabla(p-\tilde{p})\right. \\
& \left.\qquad \zeta_{t}+u^{h} \cdot \nabla \zeta+\nabla\left(\tilde{p}-p^{h}\right)\right)_{Q} \mid
\end{aligned}
$$

$$
\begin{aligned}
& \leqslant \frac{1}{8}\|\hat{\zeta}\|^{2}+C\left(h^{-1}\|\eta\|_{Q}^{2}+\sum_{m=1}^{M}\left|\eta_{-}\right|_{m}^{2}+h\left\|\eta_{t}+u^{h} \cdot \nabla \eta+\nabla(p-\tilde{p})\right\|_{Q}^{2}\right) \\
& \leqslant \frac{1}{8}\|\hat{\zeta}\|^{2}+C h^{2 k+1}+h\left\|\eta_{t}+u^{h} \cdot \nabla \eta+\nabla(p-\tilde{p})\right\|_{Q}^{2} .
\end{aligned}
$$

Similarly,

$$
\begin{aligned}
\| \eta_{t} & +u^{h} \cdot \nabla \eta+\nabla(p-\tilde{p}) \|_{Q} \\
& \leqslant\left\|\eta_{t}\right\|_{Q}+\|\nabla(p-\tilde{p})\|_{Q}+\left\|\left(u-u^{h}\right) \cdot \nabla \eta\right\|_{Q}+\|u \cdot \nabla \eta\|_{Q} \\
& \leqslant\|\eta\|_{1, Q}+\|\nabla(p-\tilde{p})\|_{Q}+\|\nabla \eta\|_{\infty}\left(\|\eta\|_{Q}+\|\zeta\|_{Q}\right) \\
& \leqslant C h^{k}+C\|\zeta\|_{Q} .
\end{aligned}
$$

Also,

$$
\begin{aligned}
\left|T_{2}\right|= & \left|\left(\left(u-u^{h}\right) \cdot \nabla u, \zeta+h\left(\zeta_{t}+u^{h} \cdot \nabla \zeta+\nabla\left(\tilde{p}-p^{h}\right)\right)\right)\right| \\
\leqslant & C\|\nabla u\|_{\infty}\|\zeta\|_{Q}\left(\|\zeta\|_{Q}+\|\eta\|_{Q}\right)+\frac{1}{8}\|\zeta\|^{2} \\
& +C h\|\nabla u\|_{\infty}\left(\|\zeta\|_{Q}+\|\eta\|_{Q}\right) .
\end{aligned}
$$

Combining (3.11) to (3.14), and using Lemma 3.1, we finally get

$$
\|\hat{\xi}\|^{2} \leqslant C\left(h^{2 k+1}+\sum_{m=1}^{M}\left|\zeta_{-}\right|_{m}^{2} h\right),
$$


so that by a discrete Gronwall inequality,

$$
\|\hat{\zeta}\|^{2} \leqslant C h^{2 k+1},
$$

from which the desired estimate follows.

Remark 3.1. From (3.7) and the velocity estimates of Theorem 3.1, we obtain the following estimate for the pressures,

$$
\left\|\nabla p-\nabla p^{h}\right\|_{Q} \leqslant C h^{k-1 / 2} .
$$

Let us now relax the $C^{1}$-condition (3.2) and assume only that $\stackrel{\circ}{\Psi}_{h} \subset \mathscr{H}$, as in Section 2, and let $W_{h}$ and $Q_{h}$ be given by (3.1) and (3.3). In this case, the discrete velocities $v \in W_{h}$ have continuous normal components across interelement boundaries, while the tangential components may be discontinuous. The natural extension of the method (3.4) to this case reads: Find $\left(u^{h}, p^{h}\right) \in W_{h} \times Q_{h}$ such that

$$
\begin{aligned}
\left(u_{t}^{h}\right. & \left.+\beta \cdot \nabla u^{h}+\nabla p^{h}, v+h\left(v_{t}+\beta \cdot \nabla v+\nabla q\right)\right)_{Q} \\
& +\sum_{K} \int_{\partial K_{-}(\beta)}\left[u^{h}\right] v_{+}\left|n_{t}+n \cdot \beta\right| d s \\
& =\left(g, v+h\left(v_{t}+\beta \cdot \nabla v+\nabla q\right)\right)_{Q} \quad \forall(v, q) \in W_{h} \times Q_{h},
\end{aligned}
$$

where $\beta=u^{h}$ and $u_{-}^{h}=u_{0}$ on $\Omega \times\{0\}$. The analysis of this method is similar to the analysis of (3.4) just presented, with a modification analogous to that presented for the Discontinuous Galerkin method (2.17), and we obtain for the method (3.15) a result analogous to that stated in Theorem 3.1, assuming now that $u \in W_{\infty}^{k+1}(Q)$.

3.2. SD-Methods for Navier-Stokes Equations. We first generalize the method (3.4) to the Navier-Stokes equations with small viscosity $\varepsilon>0$ :

$$
\begin{aligned}
u^{h}+u \cdot \nabla u+\nabla p-\varepsilon \Delta u & =g & & \text { in } \Omega \times I, \\
\operatorname{div} u & =0 & & \text { in } \Omega \times I, \\
u & =0 & & \text { on } \Gamma \times I, \\
u & =u_{0} & & \text { in } \Omega \text { for } t=0 .
\end{aligned}
$$

One is naturally led to the following method: Find $\left(u^{h}, p^{h}\right) \in W_{h} \times Q_{h}$ such that

$$
B_{\varepsilon}\left(u^{h} ; \hat{u}^{h}, \hat{v}\right)=L(\hat{v}) \quad \forall \hat{v}=(v, q) \in W_{h} \times Q_{h},
$$

where

$$
B_{\varepsilon}(w, \hat{v}, \hat{\phi})=B(w ; \hat{v}, \hat{\phi})+\varepsilon(\nabla v, \nabla \phi)_{Q}-\varepsilon \delta\left(\Delta v, \phi_{t}+u^{h} \cdot \nabla \phi+\nabla q\right)_{Q},
$$

and where $B$ and $L$ are defined by (3.5), and $\delta=\bar{C} h$ with $\bar{C}$ as specified in Lemma 3.2 below if $\varepsilon<h$, and $\delta=0$ if $\varepsilon \geqslant h$. For problem (3.16) we have the following stability result (cf. [10], [16]).

LEMMA 3.2. If $\bar{C}>0$ is sufficiently small, then for all $\hat{v} \in W_{h} \times Q_{h}$,

$$
B_{\varepsilon}\left(u^{h} ; \hat{v}, \hat{v}\right) \geqslant C\left(\|\hat{v}\|^{2}+\varepsilon\|\nabla v\|_{Q}^{2}\right),
$$

where ||$|\cdot| \mid$ is defined by (3.8).

Proof. We have for $\varepsilon>h$

$$
B_{\varepsilon}\left(u^{h} ; \hat{v}, \hat{v}\right)=\|v\|^{2}+\varepsilon\|\nabla v\|_{Q}^{2}-\varepsilon \delta\left(\Delta v, v_{t}+u^{h} \cdot \nabla v+\nabla q\right)_{Q},
$$


and by the inverse estimate

$$
\|\Delta v\|_{Q} \leqslant C h^{-1}\|\nabla v\|_{Q} \quad \forall v \in W_{h},
$$

we get

$$
\begin{aligned}
\left|\varepsilon \delta\left(\Delta v, v_{t}+u^{h} \cdot \nabla v+\nabla q\right)_{Q}\right| & \leqslant \frac{1}{2}\|\hat{v}\|^{2}+\varepsilon \delta C h^{-2} \varepsilon\|\nabla v\|_{Q}^{2} \\
& \leqslant \frac{1}{2}\left(\|\hat{v}\|^{2}+\varepsilon\|\nabla v\|_{Q}^{2}\right)
\end{aligned}
$$

if $\varepsilon \delta C h^{-2} \leqslant \frac{1}{2}$, i.e., if $\bar{C} C \leqslant \frac{1}{2}$, which proves the desired result.

Arguing as above, we obtain in the case $\varepsilon<h$ a direct analogue of Theorem 3.1 for the problem (3.17), with an additional term $\sqrt{\varepsilon}\left\|\nabla\left(u-u^{h}\right)\right\|_{Q}$ on the left-hand side.

Let us finally also consider an extension of the method (3.15) to the Navier-Stokes equations (3.16) with $W_{h}$ and $Q_{h}$ given by (3.1) and (3.3). Since the velocities $v \in W_{h}$ may be discontinuous in space, we are led to introduce an additional variable to handle the elliptic term $-\varepsilon \Delta u$. As additional variable we shall use the vorticity $\omega=$ rot $u$ and seek an approximate vorticity in the space $Q_{h}$ given by (3.3).

To motivate the formulation of the discrete problem, we first note that the Stokes problem corresponding to (3.16), obtained by omitting the nonlinear term $u \cdot \nabla u$, can, at least formally, be given the following variational formulation: Find $(u, \omega)$ : $I \rightarrow W \times Y$, where $W=\left\{\operatorname{rot} \phi: \phi \in H_{0}^{1}(\Omega)\right\}$ and $Y=H^{1}(\Omega)$, such that $u(t)=u_{0}$ for $t=0$ and on $I$,

$$
\begin{gathered}
\left(u_{t}, v\right)_{\Omega}+\varepsilon(\operatorname{rot} \omega, v)_{\Omega}=(g, v)_{\Omega} \quad \forall v \in W, \\
\varepsilon(\operatorname{rot} \theta, u)_{\Omega}=\varepsilon(\omega, \theta)_{\Omega} \quad \forall \theta \in Y .
\end{gathered}
$$

This follows from the fact that with $\omega=\operatorname{rot} u$, we have $\operatorname{rot} \omega=-\Delta u$, since $\operatorname{div} u=0$. Further, since $u=\operatorname{rot} \psi$ for some $\psi \in H_{0}^{1}(\Omega)$, we have that $u \cdot n=0$ on $\Gamma$, and (3.18b) implies that also the tangential component of $u$ vanishes on $\Gamma$, so that in fact $u=0$ on $\Gamma$.

Returning now to the Navier-Stokes equations (3.16), and handling the nonlinear term as in the case of Euler's equations, we are led to the following SD-method for (3.16): Find $\left(u^{h}, p^{h}, \omega^{h}\right) \in W_{h} \times Q_{h} \times Q_{h}$ such that

$$
\begin{aligned}
\left(u_{t}^{h}+\beta\right. & \left.\cdot \nabla u^{h}+\nabla p^{h}, v+\delta\left(v_{t}+\beta \cdot \nabla v+\nabla q\right)\right) \\
& +\sum_{K} \int_{\partial K_{-}(\beta)}\left[u^{h}\right] v_{+}\left|n_{t}+n \cdot \beta\right| d s \\
& +\varepsilon\left(\operatorname{rot} \omega^{h}, v\right)+\varepsilon \delta\left(\operatorname{rot} \omega^{h}, v_{t}+\beta \cdot \nabla v+\nabla q\right) \\
= & \left(g, v+\delta\left(v_{t}+\beta \cdot \nabla v+\nabla q\right)\right) \quad \forall(v, q) \in W_{h} \times Q_{h},
\end{aligned}
$$

$$
\varepsilon\left(\operatorname{rot} \theta, u^{h}\right)=\varepsilon\left(\omega^{h}, \theta\right) \quad \forall \theta \in Q_{h},
$$

where $\delta=\bar{C} h$ with $\bar{C}>0$ sufficiently small and, as before, $\beta=u^{h}$ and $u_{-}^{h}(\cdot, 0)=u_{0}$. Choosing $(v, q, \theta)=\left(u^{h}, p^{h}, \omega^{h}\right)$ in (3.19), we obtain as above the following stability estimate:

$$
\begin{aligned}
\left|u^{h}\right|_{M}^{2} & +\left|u^{h}\right|_{0}^{2}+\sum_{K} \int_{\partial K_{-}(\beta)^{\prime}}\left[u^{h}\right]^{2}\left|n_{t}+\beta \cdot n\right| d s \\
& +\delta\left\|u_{t}^{h}+\beta \cdot \nabla u^{h}+\nabla p^{h}\right\|_{Q}^{2}+\varepsilon\left\|\omega^{h}\right\|_{Q}^{2} \\
\leqslant & C\left(\|f\|_{Q}+\left\|u_{0}\right\|_{\Omega}\right),
\end{aligned}
$$


from which the following error estimate can be derived, following the proof of Theorem 3.1:

$$
\max _{0 \leqslant t \leqslant T}\left\|u(\cdot, t)-u^{h}(\cdot, t)\right\|_{\Omega}+h\left\|\nabla p-\nabla p^{h}\right\|_{Q}+\sqrt{\varepsilon}\left\|\omega-\omega^{h}\right\|_{Q} \leqslant C h^{k+1 / 2},
$$

assuming that $\varepsilon<h$ and

$$
\|u\|_{k+1, \infty}+\|p\|_{k+1}+\|\omega\|_{k+1}<\infty .
$$

Remark 3.2. The approximability properties of the space $Q_{h}$ for the vorticity $\omega^{h}$ are somewhat better than needed; it would be sufficient to have $\|\omega-\tilde{\omega}\| \leqslant C h^{k+1 / 2}$ with $\tilde{\omega} \in Q_{h}$ an interpolant of $\omega$.

4. Appendix. As an example of a uniqueness result for the methods considered, let us prove that the solutions of the discrete problem (2.6) are unique if the corresponding exact solution $\omega$ is smooth enough, i.e., satisfies (2.16), and $h$ is small enough. Thus, suppose that $\omega^{h}$ and $\bar{\omega}^{h}$ are two solutions of (2.6) with corresponding velocities $u^{h}\left(\omega^{h}\right)$ and $\bar{u}^{h}\left(\bar{\omega}^{h}\right)$. By subtraction we then have for any $m$, writing $\theta=\omega^{h}-\bar{\omega}^{h}$ and assuming that $\theta\left(\cdot, t_{m}\right)_{-}=0$,

$$
\begin{aligned}
h(f, & \left.\left(u^{h}\left(\omega^{h}\right)-\bar{u}^{h}\left(\bar{\omega}^{h}\right)\right): \nabla \theta\right)_{m} \\
= & \left(\theta_{t}, \theta\right)_{m}+\left|\theta_{+}\right|_{m}^{2}+\left(u^{h}\left(\omega^{h}\right) \cdot \nabla \theta, \theta\right)_{m} \\
& +\left(\left(\bar{u}^{h}\left(\bar{\omega}^{h}\right)-u^{h}\left(\omega^{h}\right)\right) \cdot \nabla \bar{\omega}^{h}, \theta+h\left(\theta_{t}+u^{h}\left(\omega^{h}\right) \cdot \nabla \theta\right)\right)_{m} \\
& +h\left\|\theta_{t}+u^{h}\left(\omega^{h}\right) \cdot \nabla \theta\right\|_{m}^{2} \\
& +h\left(\bar{\omega}_{t}^{h}+\bar{u}^{h}\left(\bar{\omega}^{h}\right) \cdot \nabla \bar{\omega}^{h},\left(\bar{u}^{h}\left(\bar{\omega}^{h}\right)-u^{h}\left(\omega^{h}\right)\right) \cdot \nabla \theta\right)_{m} .
\end{aligned}
$$

By Theorem 2.1 and inverse estimates it is easy to see that

$$
h^{\alpha}\|\bar{\omega}\|_{1, \infty}+\left\|\bar{u}^{h}\left(\bar{\omega}^{h}\right)\right\|_{\infty} \leqslant C,
$$

with $\alpha=\frac{1}{2}$ if $k=1$ and $\alpha=0$ if $k \geqslant 2$. Together with (4.1), this proves that

$$
\left|\theta_{-}^{2}\right|_{m+1}+\left|\theta_{+}\right|_{m}^{2}+h\left\|\theta_{t}+u^{h}\left(\omega^{h}\right) \cdot \nabla \theta\right\|_{m}^{2} \leqslant C h^{-\alpha}\|\theta\|_{m}^{2},
$$

by virtue of the fact that

$$
\left\|\bar{u}^{h}\left(\bar{\omega}^{h}\right)-u^{h}\left(\omega^{h}\right)\right\|_{m} \leqslant C\|\theta\|_{m} .
$$

However, by the argument used in the proof of Lemma 3.1, we have

$$
\|\theta\|_{m}^{2} \leqslant C\left[h^{2}\left\|\theta_{t}+u^{h}\left(\omega^{h}\right) \cdot \nabla \theta\right\|_{m}^{2}+h\left|\theta_{+}\right|_{m}^{2}\right],
$$

and thus

$$
h^{-1}\|\theta\|_{m}^{2} \leqslant C h^{-\alpha}\|\theta\|^{2},
$$

which shows that $\theta \equiv 0$ if $h$ is small enough, and the uniqueness result follows.

Department of Mathematics

Chalmers University of Technology

41296 Göteborg, Sweden

Department of Mathematics

Faculty of Technology

University of Oulu

95070 Oulu, Finland 
1. J. T. Beale \& A. Majda, "Vortex methods. I and II," Math. Comp., v. 39, 1982, pp. 1-27 and 29-52.

2. P. G. Ciarlet, The Finite Element Method for Elliptic Problems, North-Holland, Amsterdam, 1978.

3. K. Eriksson, C. Johnson \& V. ThOmEe, "Time discretization of parabolic problems by the discontinuous Galerkin method," Math. Modelling and Numer. Anal., v. 19, 1985, pp. 611-643.

4. V. Girault \& P. A. Raviart, "An analysis of a mixed finite element method for the Navier-Stokes equations," Numer. Math., v. 33, 1979, pp. 235-271.

5. T. J. Hughes \& A. Brooks, "A multidimensional upwind scheme with no crosswind diffusion," in AMD, v. 34, Finite Element Methods for Convection Dominated Flows (T. J. Hughes, ed.), ASME, New York, 1979.

6. T. J. Hughes \& A. Brooks, "A theoretical framework for Petrov-Galerkin methods with discontinuous weighting functions: Application to the streamline-upwind procedure," Finite Elements in Fluids, Vol. 4 (R. H. Gallagher, ed.), Wiley, New York, 1982.

7. T. J. Hughes, E. T. Tezduyar \& A. Brooks, Streamline Upwind Formulation for Advection-Diffusion. Navier-Stokes and First Order Hyperbolic Equations, Fourth Internat. Conf. on Finite Element Methods in Fluids, Tokyo, July, 1982.

8. C. JOHNSON \& U. NÄVERT, "An analysis of some finite element methods for advection-diffusion," in Analytical and Numerical Approaches to Asymptotic Problems in Analysis (O. Axelsson, L. S. Frank and A. Van der Sluis, eds.), North-Holland, Amsterdam, 1981.

9. C. JoHnson, "Finite element methods for convection-diffusion problems," in Computing Methods in Engineering and Applied Sciences. V (R. Glowinski and J. L. Lions, eds.), North-Holland, Amsterdam, 1981 .

10. C. Johnson, U. Nävert \& J. Pitkäranta, "Finite element methods for linear hyperbolic problems," Comput. Methods Appl. Mech. Engrg., v. 45, 1985, pp. 285-312.

11. C. Johnson \& J. PitkÄranta, "An analysis of the discontinuous Galerkin method for a scalar hyperbolic equation," Math. Comp., v. 46, 1986, pp. 1-26.

12. C. Johnson, Error Estimates and Automatic Time Step Control for Numerical Methods for Stiff Ordinary Differential Equations, Technical report, Chalmers Univ. of Technology, Göteborg, 1984.

13. P. Lesaint, Sur la Résolution des Systèmes Hyperboliques du Premier Ordre par des Méthodes d'Elements Finis, Thèse, Université Paris VI, 1975.

14. P. Lesaint \& P. A. Raviart, "On a finite element method for solving the neutron transport equation," in Mathematical Aspects of Finite Elements in Partial Differential Equations (C. de Boor, ed.), Academic Press, New York, 1974, pp. 89-123.

15. J. L. Lions, Quelques Méthodes de Résolution des Problèmes aux Limites non Linéaires, Dunod, Paris, 1969.

16. U. Nävert, A Finite Element for Convection-Diffusion Problems, Thesis, Chalmers Univ. of Technology, Göteborg, 1982.

17. R. Tемам, "Local existence of $C^{x}$ solutions of the Euler equations of incompressible perfect fluids," in Turbulence and Navier Stokes Equations (R. Temam, ed.), Lecture Notes in Math., Vol. 565. Springer, Berlin and New York, 1976. 